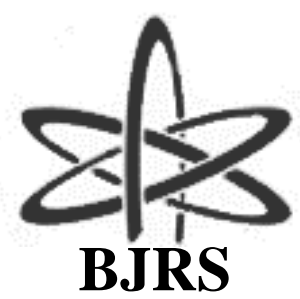

BRAZILIAN JOURNAL

$\mathrm{OF}$

RADIATION SCIENCES

08-01A (2020) 01-12

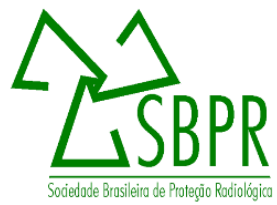

\title{
Análise de risco de exposição potencial em medidor nuclear de nível de bebidas
}

\author{
Elbern $^{a}$ M.K., Souto ${ }^{a}$ E.B., Souto ${ }^{a}$ G.D.B. \\ a Pro-Rad Consultores em Radioproteção S/S Ltda., 94910-000, Cachoeirinha, RS, Brasil \\ martin@prorad.com.br
}

\section{RESUMO}

Recentemente a CNEN tem requerido análise das exposições potenciais para medidores nucleares de nível de bebidas. Portanto, urge a necessidade de o profissional de proteção radiológica saber realizar a análise das condições de risco a fïm de verificar a necessidade de ações adicionais para evitar ou limitar a exposição potencial. Para qualificar a magnitude dos riscos ocupacionais frente ao agente de risco ambiental ocupacional Radiação Ionizante, aplicou-se o método conhecido como Análise Preliminar de Risco (APR). Para uma análise de risco mais completa o expert deve conhecer, o mais possível, os muitos requisitos e critérios a serem atendidos, como regulamentações trabalhistas, sanitárias, ambientais, etc. Neste estudo, para esta magnitude de risco, não houve necessidade de ação adicional.

Palavras chave: Análise de Risco, Classificação de área, Exposição potencial, Radiação Ionizante

\section{ABSTRACT}

Recently, CNEN has been requiring analysis of potential exposures for beverage nuclear gauges. Therefore, there is a need for the radiation protection professional to be able to analyze risk conditions in order to verify the need for additional actions to avoid or limit potential exposures. To qualify the magnitude of occupational hazards against the occupational environmental risk agent Ionizing Radiation, the method known as Preliminary Hazard Analysis (PHA) was applied. For a more complete risk analysis the expert should know as much as possible the requirements and criteria to be met, such as labor, health, environmental, etc. In this study, for this magnitude of risk, there was no need for further action.

Keywords: Risk analysis, Area classification, Potential exposure, Ionizing radiation. 


\section{INTRODUÇÃO}

Recentemente a CNEN tem requerido análise das exposições potenciais para medidores nucleares de nível de bebidas, com solicitações similares ao texto a seguir [1].

A saber, deve ser classificada como área controlada qualquer área na qual medidas específicas de proteção radiológica são ou podem ser necessárias para: a) Controlar as exposições de rotina e evitar a disseminação da contaminação durante as condições normais de operação; ou b) Evitar ou limitar a extensão das exposições potenciais. A antiga Norma CNEN-NN-3.01 utilizava para classificação de áreas apenas os níveis de exposição ocupacional quantificados pelos valores de taxas de dose. A Norma CNEN-NN-3.01 foi atualizada e o conceito de área controlada foi ampliado, de modo que a classificação de áreas agora deve levar em conta tanto os níveis normais de exposição normais como também a prevenção ou redução da magnitude das exposições potenciais.

Embora, em algumas práticas, as exposições normais esperadas sejam baixas, há a probabilidade de exposições potenciais, principalmente nos casos de acesso dos indivíduos às fontes na linha de produção. Sendo assim, a instalação deve avaliar sua classificação de área considerando a possibilidade de indivíduos serem expostos. É necessário também que seja especificado no Plano de Radioproteção como será restringido o acesso dos indivíduos do público às fontes de radiação de forma a diminuir a probabilidade de ocorrência de exposições potenciais.

Portanto, urge a necessidade de o profissional de proteção radiológica saber realizar a análise das condições de risco a fim de verificar a necessidade de ações específicas para evitar ou limitar a exposição potencial. Este trabalho tem por objetivo apresentar uma metodologia para avaliação do risco de exposição potencial em medidores nucleares utilizados para controle de nível de bebidas.

O termo exposição potencial é utilizado para uma exposição cuja ocorrência não é uma certeza. Eventos incomuns nas operações e condições ambientais podem resultar em doses anuais acima dos limites de operação normais. Exposições que podem ocorrer em função desses eventos são denominadas exposições potenciais. [2] 


\section{MATERIAIS E MÉTODOS}

O processo de gerenciamento de riscos, como todo procedimento de tomada de decisão, começa com a identificação e análise de um problema. Não existe um método ótimo para se identificar riscos. O risco é uma relação entre dano potencial e a medida de proteção utilizada. O risco pode ser quantificado ou também pode ser não quantificável. A parcela de risco não quantificável é sempre tratada de forma subjetiva conforme critério e experiência de cada profissional. Ao se gerenciar riscos é importante saber o critério para se considerar um risco como aceitável ou inaceitável [2]. Neste estudo de casos os critérios foram os limites de dose anual da legislação brasileira [3] e o tipo de efeito biológico [4, 5].

Os dois conceitos mais positivos e amplamente utilizados para a elevação do nível de segurança são o conceito de ALARA e da cultura de segurança. ALARA está intimamente ligada ao processo de otimização. O objetivo é que o detrimento causado por uma prática seja reduzido de tal forma que outras reduções se tornem menos importantes que o esforço adicional para serem executadas.

Risco não é a probabilidade de ocorrer uma exposição à radiação, mas sim de ocorrer um efeito indesejado após uma exposição. O risco individual pode ser expresso em termos do produto da probabilidade da exposição e da probabilidade do efeito indesejado resultante desta exposição [2]. A análise de exposições potenciais busca evitar que ocorra exposição ao agente capaz de causar dano. Portanto, limites de dose não se aplicam às exposições potenciais, pois, antes ou após análise de risco, são implementadas salvaguardas que as tornam extremamente improváveis. Ou seja: o objetivo é que a exposição potencial não ocorra; não é limitar a dose da exposição. [2]

A Agência Internacional de Energia Atômica (AIEA) [6], informa que existem diversos métodos estabelecidos para análises de acidentes e apresenta uma introdução ao método da Árvore de Eventos, o qual pode ser qualitativo ou quantitativo, se for possível estimar as probabilidades de falhas [6].

Para uma análise de riscos também se faz necessário saber as características do trabalho a ser executado: tipo e probabilidade da exposição, estimativa da dose total a ser recebida, procedimentos utilizados para manter as doses ALARA, restrições da empresa e da legislação local, informações relevantes do serviço de radioproteção como auditorias periódicas, restrições de dose e limites 
operacionais, etc. Para analisar riscos não quantificáveis, o método mais amplamente utilizado é a Análise Preliminar de Risco (APR) [7].

\subsection{Análise Preliminar de Risco}

Para qualificar a magnitude dos riscos ocupacionais frente ao agente de risco ambiental ocupacional Radiação Ionizante, aplicou-se o método conhecido como APR. Trata-se de uma metodologia amplamente aplicada em segurança do trabalho. O risco é classificado por uma relação entre magnitude e probabilidade e, em função dessa classificação, são definidas ações a serem tomadas. Uma matriz de análise de risco realiza o cruzamento das informações de severidade (magnitude) do efeito danoso e probabilidade (frequência) de o efeito ocorrer [7], vide tabela 1.

Tabela 1: Matriz de classificação de risco.

\begin{tabular}{cccccc}
\hline \multirow{2}{*}{ Severidade } & \multicolumn{3}{c}{ Frequência } \\
\cline { 2 - 6 } & $\begin{array}{c}\text { Extremamente } \\
\text { Remota }\end{array}$ & Remota & Improvável & Provável & Frequente \\
\hline Catastrófica & Menor & Moderado & Sério & Crítico & Crítico \\
Crítica & Desprezível & Menor & Moderado & Sério & Crítico \\
Marginal & Desprezível & Desprezível & Menor & Moderado & Sério \\
Desprezível & Desprezível & Desprezível & Desprezível & Menor & Moderado \\
\hline
\end{tabular}

OBS: As normas técnicas não definem um modelo específico de APR. Cada empresa ou expert pode desenvolver o modelo de APR que melhor atenda suas necessidades de segurança.

Uma vez classificado o risco se deve, em função da sua magnitude, definir as proteções a serem utilizadas, se as proteções existentes são suficientes e se são necessárias ações adicionais de proteção radiológica para evitar ou limitar a exposição potencial. Se o risco for desprezível não há necessidade de ações adicionais de proteção radiológica; caso contrário, significa que devem ser tomadas ações para reduzir o risco: seja aumentando a proteção, seja reduzindo o efeito danoso. Estas ações variam desde ações administrativas, como treinamentos, diálogos diários de segurança (DDS), auditorias e limitação de acesso (incluindo classificação de áreas), até soluções de engenharia, como equipamentos de proteção coletiva. 


\subsection{Classificação de áreas}

A norma CNEN NN 3.01 [3] não teve mudanças no seu item 5.8 (classificação de áreas) desde sua publicação através da Resolução CNEN 27/4 de 06/01/2005. Entre as alterações posteriores a 2005, nenhuma se aplica a classificação de área (foram alterados os itens: 1.2.5, 2.2, 5.4.2.1, 5.4.3.1, 5.4.3.4 e 7). Portanto, no ano de 2019, não se deveria escrever "A Norma CNEN-NN-3.01 foi atualizada e o conceito de área controlada foi ampliado, de modo que a classificação de áreas agora deve levar em conta tanto os níveis normais de exposição normais como também a prevenção ou redução da magnitude das exposições potenciais.” em um ofício [1]. Desde 2005 são levadas em conta as exposições potenciais. Esta análise não costuma constar nos planos de radioproteção, pois, conforme item 5.4.3.3, é um processo de otimização cuja demonstração é dispensável.

A norma CNEN NN 3.01/PR004:2011 [8], em seu item 3.3, estabelece: "Fora das áreas designadas como controladas ou supervisionadas, a taxa de dose e o risco de contaminação por materiais radioativos devem ser baixos o suficiente para assegurar que, em condições normais, o nível de proteção para aqueles que trabalham no local seja comparável com o nível de proteção requerido para exposições do público. Tais áreas são denominadas áreas livres, do ponto de vista de proteção radiológica ocupacional." Medidores nucleares de nível de bebidas utilizam fontes de raios $\mathrm{X}$ de $60 \mathrm{keV}$ ou fontes seladas de Am-241. Em equipamentos de raios X não existe risco de contaminação. Para fontes seladas, devido ao processo de fabricação, é necessário apenas o controle externo da dose de radiação.

\section{ANÁLISE DO RISCO}

Para analisar o risco se deve elencar os possíveis perigos (efeitos indesejados), suas magnitudes (severidade) e suas probabilidades (frequência) de ocorrência [7].

Para as circunvizinhanças da área de irradiação, conforme levantamentos radiométricos realizados pelo servico de radioproteção na posição mais próxima, a cerca de $5 \mathrm{~cm}$, das fontes de raios $\mathrm{X}$ de $60 \mathrm{keV}$ e Am-241 de $100 \mathrm{mCi}$, os níveis de radiação apresentaram valores de radiação natural de fundo. Para avaliar as fontes citadas, em caso de exposição potencial, foram utilizados 
detectores TLD de LiF:Mg,Ti para medir as taxas de dose na saída do feixe dos medidores de nível de bebidas de 2 empresas diferentes. Em cada avaliação foram utilizados dez conjuntos contendo três detectores TLD envoltos em plástico, posicionados em frente e envolta do ponto de entrada do feixe no detector do medidor de nível. A cada avalliação, foi estabelecido o tempo mínimo de 1 hora e o tempo exato foi cronometrado para possiblitar o cálculo da taxa de dose. As maiores taxas de dose mensuradas foram de 6,5 mSv/h, na empresa 1; e 9,0 mSv/h na empresa 2.

A exposição ao feixe de radiação só é possível em caso de falha de procedimento de manutenção ou por exposição intencional. Nesses casos, a área irradiada deve, conforme figuras $1 \mathrm{e}$ 2, devido ao espaço disponível, ser uma extremidade do corpo, cujo limite de tolerância [3] é maior do que para o corpo inteiro (limite de extremidade para indivíduo ocupacionalmente exposto - IOE; ou para pele de indivíduo do público - IP).

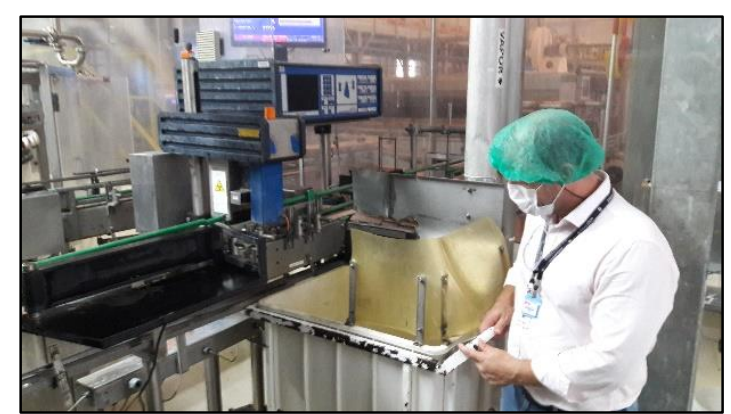

Figura 1: Medidores nucleares para medição de nível em bebidas, empresa 1. Fonte: arquivo pessoal
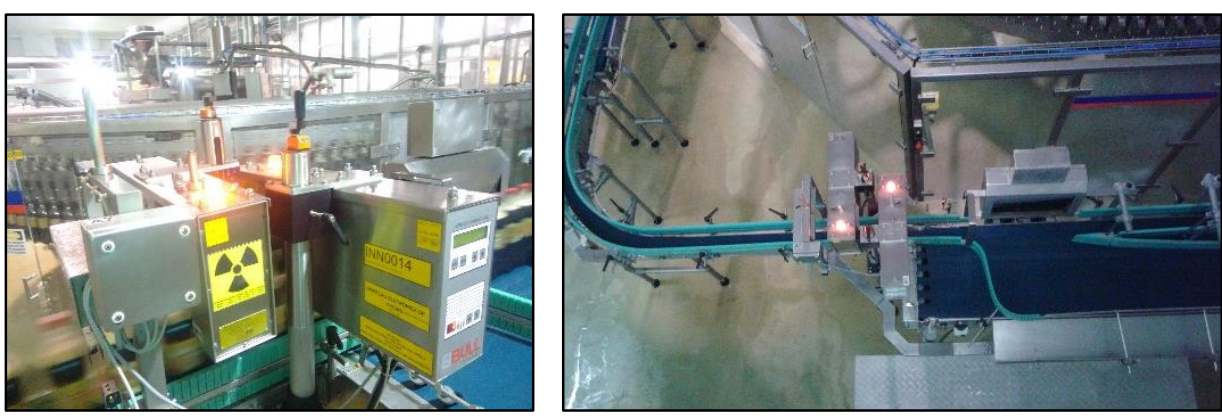

Figura 2: Medidores nucleares para medição de nível em bebidas, empresa 2. Fonte: arquivo pessoal 
Estabeleceu-se uma lista de efeitos indesejados, severidades e frequências para classificar o risco conforme a matriz apresentada na metodologia.

A tabela 2 apresenta os tempos de exposição necessários para a ocorrência de possíveis efeitos e doses, considerando uma jornada laboral de 8 horas por dia (2000 horas por ano), e a tabela 3 os critérios utilizados classificar a severidade do efeito indesejado. Nos casos de probabilidade de câncer, estas foram calculadas conforme Tauhata et al [5].

Tabela 2: Tempos de exposição necessário à ocorrência de possíveis efeitos e limites de dose

\begin{tabular}{cccc}
\hline \multirow{2}{*}{ Efeito ou limite de dose } & \multirow{2}{*}{ Dose } & \multicolumn{2}{c}{$\begin{array}{c}\text { Tempo de exposição necessário } \\
\text { junto ao detector }\end{array}$} \\
\cline { 3 - 4 } & & $\mathbf{6 , 5} \mathbf{~ m S v} / \mathbf{h}$ & $\mathbf{9 , 0} \mathbf{~ m S v} / \mathbf{h}$ \\
\hline Limite anual para corpo inteiro de IP & $1,0 \mathrm{mSv}$ & $9,2 \mathrm{~min}$ & $6,7 \mathrm{~min}$ \\
Intervenção, limite genérico para IP* & $10 \mathrm{mSv}$ & $1 \mathrm{~h} 32 \mathrm{~min}$ & $1 \mathrm{~h} 6 \mathrm{~min}$ \\
Limite anual para cristalino de IP & $15 \mathrm{mSv}$ & $2 \mathrm{~h} 18 \mathrm{~min}$ & $1 \mathrm{~h} 40 \mathrm{~min}$ \\
Limite anual para pele de IP** & $50 \mathrm{mSv}$ & $769 \mathrm{~h} 13 \mathrm{~min}$ & $555 \mathrm{~h} 33 \mathrm{~min}$ \\
Limite anual para corpo inteiro de IOE & $50 \mathrm{mSv}$ & $7 \mathrm{~h} 40 \mathrm{~min}$ & $5 \mathrm{~h} 33 \mathrm{~min}$ \\
Limite para emergências & $100 \mathrm{mSv}$ & $15 \mathrm{~h} 23 \mathrm{~min}$ & $11 \mathrm{~h} 6 \mathrm{~min}$ \\
Limite anual para extremidades de IOE & $500 \mathrm{mSv}$ & $>3,8$ anos & $>2,7$ anos \\
Eritema* & $3.000 \mathrm{mGy}$ & $>23$ anos & $>16$ anos \\
\hline
\end{tabular}

* Vide [9], **considerando Wt [10], OBS: Limites de dose não são efeitos.

Note-se que os tempos de exposição calculados na tabela 2 são incompatíveis com a parada de linhas de produção que avaliam o nível em dezenas de milhares de latas ou garrafas de bebida por hora.

Tabela 3: Critérios para classificação da severidade do efeito indesejado

\begin{tabular}{ll}
\hline Severidade & Critério \\
\hline Catastrófica & Pode levar a morte \\
Crítica & Efeito determinístico \\
Marginal & Efeito estocástico causado por dose acima do limite de exposição \\
Desprezível & Efeito estocástico causado por dose menor ou igual ao limite de exposição \\
\hline
\end{tabular}

Após a análise de risco, se deve avaliar as proteções existentes para verificar se são necessárias ações adicionais de proteção radiológica. Em ambas as empresas, as principais condições de segurança existentes são: 
- Não há postos de trabalho próximo das fontes de radiação;

- Não há circulação corriqueira de pessoas durante a operação normal;

- A blindagem dos medidores nucleares de nível é proveniente de fábrica e é eficiente para reduzir a taxa de dose para abaixo do limite para área livre;

- Existe sinalização dos medidores nucleares com trifólio e texto indicativo de fonte de radiação para advertir indivíduos do público e IOE;

- Os levantamentos radiométricos periódicos confirmam a classificação de área livre;

- Os trabalhadores do Serviço Especializado em Engenharia de Segurança e em Medicina do Trabalho (SESMT) [9] que estão diretamente envolvidos com a proteção radiológica são IOE.

- Há treinamento anual para IOE;

- O serviço de proteção radiológica é auditado por supervisor de proteção radiológica;

- As fontes de radiação, conforme padrão de segurança da AIEA, são da classe de risco de categoria 5, ou seja: são fontes consideradas improváveis de causarem severos efeitos danosos [6].

\section{RESULTADOS E DISCUSSÃO}

O resultado é apresentado na tabela 4. Em todos os casos, a mínima ação necessária é realizar controle da exposição ocupacional normal conforme legislação Trabalhistas $[11,12,13]$ e da CNEN [3]. Para fins de comparação também foram analisadas situações de exposição de corpo inteiro.

Tabela 4: Análise e classificação do risco.

\begin{tabular}{cccccc}
\hline Descrição & Efeito & $\begin{array}{c}\text { Probabilidade } \\
\text { de câncer [4] }\end{array}$ & $\begin{array}{c}\text { Severidade } \\
\text { do efeito }\end{array}$ & $\begin{array}{c}\text { Frequência } \\
\text { do efeito }\end{array}$ & $\begin{array}{c}\text { Classificação } \\
\text { do Risco }\end{array}$ \\
\hline $\begin{array}{c}\text { Limite de dose para } \\
\text { corpo inteiro para } \\
\text { IP }(1 \mathrm{mSv})\end{array}$ & $\begin{array}{c}\text { Efeito } \\
\text { estocástico de } \\
\text { probabilidade } \\
\text { aceitável }\end{array}$ & $0,005 \%$ & Desprezível & Improvável & Desprezível \\
\hline $\begin{array}{c}\text { Limite de dose para } \\
\text { corpo inteiro para } \\
\text { IOE }(50 \mathrm{mSv})\end{array}$ & $\begin{array}{c}\text { Efeito } \\
\text { estocástico de } \\
\text { probabilidade } \\
\text { aceitável }\end{array}$ & $0,25 \%$ & Desprezível & Improvável & Desprezível \\
\hline
\end{tabular}




\begin{tabular}{|c|c|c|c|c|c|}
\hline $\begin{array}{l}\text { Pele, para IP (50 } \\
\text { mSv) }\end{array}$ & $\begin{array}{c}\text { Efeito } \\
\text { estocástico de } \\
\text { probabilidade } \\
\text { aceitável } \\
\end{array}$ & $0,0025 \%$ & Desprezível & $\begin{array}{l}\text { Extremamente } \\
\text { remota }\end{array}$ & Desprezível \\
\hline $\begin{array}{c}\text { Exigência de } \\
\text { aconselhamento } \\
\text { médico }(100 \mathrm{mSv})\end{array}$ & $\begin{array}{c}\text { Efeito } \\
\text { estocástico de } \\
\text { probabilidade } \\
\text { aceitável } \\
\end{array}$ & $0,5 \%$ & Marginal & $\begin{array}{l}\text { Extremamente } \\
\text { remota }\end{array}$ & Desprezível \\
\hline $\begin{array}{l}\text { Limite de dose para } \\
\text { extremidades para } \\
\text { IOE }(500 \mathrm{mSv})\end{array}$ & $\begin{array}{c}\text { Efeito } \\
\text { estocástico de } \\
\text { probabilidade } \\
\text { aceitável }\end{array}$ & $0,025 \%$ & Desprezível & $\begin{array}{l}\text { Extremamente } \\
\text { remota }\end{array}$ & Desprezível \\
\hline Eritema (3 Gy) & $\begin{array}{c}\text { Eritema e } \\
\text { probabilidade } \\
\text { de câncer } \\
\text { inaceitável } \\
\end{array}$ & $15 \%$ & Crítica & $\begin{array}{c}\text { Extremamente } \\
\text { remota }\end{array}$ & Desprezível \\
\hline $\begin{array}{c}\text { Dose de corpo } \\
\text { inteiro, letal em } \\
50 \% \text { dos casos não } \\
\text { tratados (4 Gy) }\end{array}$ & Morte & - & Catastrófica & $\begin{array}{l}\text { Extremamente } \\
\text { remota }\end{array}$ & Menor \\
\hline $\begin{array}{l}\text { Exposição da mão, } \\
\text { junto à saída do } \\
\text { feixe }(9 \mathrm{mSv} / \mathrm{h})\end{array}$ & $2000 \mathrm{~h} /$ ano & $0,9 \%$ & Crítica & $\begin{array}{l}\text { Extremamente } \\
\text { remota }\end{array}$ & Desprezível \\
\hline $\begin{array}{l}\text { Exposição da mão, } \\
\text { junto à saída do } \\
\text { feixe }(9 \mathrm{mSv} / \mathrm{h})\end{array}$ & 555 h/ano & $0,25 \%$ & Desprezível & $\begin{array}{l}\text { Extremamente } \\
\text { remota }\end{array}$ & Desprezível \\
\hline
\end{tabular}

IP: Individuos do público; IOE: Indivíduos ocupacionalmente expostos.

Para uma análise de risco completa se precisará ampliar o campo de visão e conhecer, o mais possível, os muitos requisitos e critérios a serem atendidos, como regulamentações trabalhistas, sanitárias, ambientais, etc. No Brasil, por exemplo, existem diversas normas regulamentadoras (NR) que se aplicam a relações de trabalho. Alguns exemplos de NRs aplicáveis à análise do risco de efeitos indesejados possíveis de serem causados por radiação ionizante estão indicadas nas referências $[11,12,13]$.

Também é importante que se perceba que existem consequências relacionadas à demarcação de uma área controlada que, durante muitos anos, se manteve classificada como área livre. Estas consequências devem ser consideradas ao se aplicar o conceito ALARA, ou seja: se o esforço 
adicional para estabelecer uma nova área controlada não se compensar com significativa redução da dose, a classificação da área não deverá mudar.

As consequências relacionadas à mudança da classificação das áreas circunvizinhas aos medidores nucleares de nível de bebidas, de livre para controlada, para os locais em análise são:

a. A dose efetiva anual dos IP não sofrerá redução significativa, permanecendo condizente com o nível de radiação natural do local.

b. A dose equivalente anual na pele dos IP não sofrerá redução significativa, permanecendo condizente como nível de radiação natural do local.

c. Radiofobia.

d. Não há histórico de acidentes nos locais avaliados, portanto uma classificação de área mais rigorosa levantará algumas dúvidas:

i. A classificação da área estava errada durante todos estes anos?

ii. Isso significa que trabalhei em área controlada durante anos?

iii. Devo processar a empresa para receber adicional de periculosidade por ter trabalhado em área controlada que estava mal classificada?

e. Um único processo de pedido de adicional de periculosidade, mais provável que qualquer exposição potencial, custará bastante caro. Além dos custos processuais e de honorários, há o valor da causa que será atribuído com base nos salários dos últimos 60 meses (5 anos), aproximadamente 60 vezes 30\% do salário base do autor da causa. Ao se multiplicar este valor pela quantidade de IP que ali transitaram, percebe-se que o custo para se estabelecer uma área controlada nestas condições é bastante elevado.

\section{CONCLUSÃO}

Risco é a relação entre o dano potencial e a medida de proteção utilizada. Quanto maior a proteção menor o risco, para o mesmo dano potencial. Quanto maior o dano potencial, tão maior deve ser a proteção para que o risco se mantenha controlado. No exemplo explorado, é extremamente improvável que uma pessoa se acidente ou se exponha intencionalmente à radiação, encostando-se na saída do feixe de radiação de um medidor de nível de bebidas. Nesta situação, 
mesmo considerando que a pessoa exporia intencionalmente sua mão ao feixe durante toda jornada laboral (2000 h/ano), o possível efeito danoso é um efeito estocástico cuja severidade está acima do limite aceitável, mas a probabilidade irrisória desta situação ocorrer torna o risco desprezível. Para a magnitude de risco: desprezível, não há necessidade de ação adicional e pode-se dizer que as exposições estão ALARA.

Calculou-se o tempo necessário para a probabilidade de efeito estocástico igualar o limite de dose ocupacional para IOE (para o qual a magnitude do risco ainda é desprezível): cerca de 555 horas, ou seja: cerca de 69 dias de trabalho, considerando jornada laboral de 8 horas. Como se pode perceber, qualquer exposição potencial levará a um tempo de exposição bastante inferior a estes 69 dias, confirmando a classificação de improvável risco de exposição potencial da AIEA [6].

Conforme a análise acima se conclui, devido à classificação do risco e a impossibilidade, na prática, de exposição acidental acima de alguns poucos minutos: não haver necessidade de se adotar medidas adicionais de proteção radiológica para controlar as exposições de rotina, evitar a disseminação da contaminação ou evitar ou limitar a extensão das exposições potenciais.

\section{REFERÊCIAS}

[1] CNEN - Comissão Nacional de Energia Nuclear. Ofício, 2019, 2p.

[2] INSAG - International Nuclear Safety Advisory Group. Potential Exposure in Nuclear Safety. INSAG-9, Vienna: IAEA, 1995. 43p.

[3] CNEN - Comissão Nacional de Energia Nuclear. Diretrizes Básicas de Proteção Radiológica. CNEN NN 3.01, 2014. 22p.

[4] ICRP - International Commission on Radiological Protection. The 2007 Recommendations of the International Commission on Radiological Protection. ICRP Publication 103, ICRP, 2007. 334p.

[5] Tauhata, L., Salati, I. P. A., Di Prinzio, R., Di Prinzio, M. A. R. R. Radioproteção e Dosimetria: Fundamentos, $10^{a}$ revisão. Rio de Janeiro, IRD/CNEN, 2014. 344p. 
[6] IAEA - International Atomic Energy Agency. Safety of Radiation Generators and sealed sources. IAEA Safety Standards RS-G-1.10, Vienna: IAEA, 2006. 71p.

[7] Portela, Geraldo. Gerenciamento de Riscos na Indústria de Petróleo e Gás - Conceitos e casos onshore e offshore, 1a. ed. Rio de Janeiro: Elsevier, 2015. 520p.

[8] CNEN - Comissão Nacional de Energia Nuclear. Restrição de dose, Níveis de referência ocupacionais e Classificação de áreas. CNEN NN 3.01 / PR 004:2011, 2011. 4p.

[9] CNEN - Comissão Nacional de Energia Nuclear. Níveis de intervenção e de ação para exposição crônica. CNEN NN 3.01 / PR 007:2005, 2005. 3p.

[10] CNEN - Comissão Nacional de Energia Nuclear. Fatores de Ponderação para as grandezas de proteção radiológica. CNEN NN 3.01 / PR 002:2011, 2011. 4p.

[11] MTPS - Ministério do Trabalho e Previdência Social. NR-4 Serviços Especializados em Engenharia de Segurança e em Medicina do Trabalho. Portaria MTPS 510, 2016, 30p.

[12] MTb - Ministério do Trabalho. NR-9 Programa de Prevenção de Riscos Ambientais. Portaria MTb 871, 2017, 13p.

[13] MTb - Ministério do Trabalho. NR-15 Atividades e Operações Insalubres. Portaria MTb n. ${ }^{\text {o }} 1.084,2018,82 \mathrm{p}$. 